\title{
Eliciting Emotion and Action Increases Social Media Engagement: An Analysis of Influential Orthopaedic Surgeons
}

\author{
Muhammad J. Abbas, B.S., Lafi S. Khalil, M.D., Abdulah Haikal, B.A., Miriam E. Dash, M.S., \\ Gauthier Dongmo, B.S., and Kelechi R. Okoroha, M.D.
}

\begin{abstract}
Purpose: The purpose of this study is to analyze the Instagram practices of current orthopaedic surgeons and the components associated with highest reach and interactions. Methods: The top 25 orthopaedic surgeon Instagram profiles using the hashtag \#ortho were ranked by the number of followers. Account information regarding followers, posts, engagement percentage, average likes, average comments, average video view, average video likes, average video comments, and estimated cost per post was recorded using social media marketing tools. An analysis of each Instagram profiles' top 10 posts, based on number of likes, was conducted. A coding framework was developed to categorized posting strategies utilized. Results: Twenty-five Instagram accounts and 250 Instagram posts were included in the analysis. Accounts with increased engagement rating had a significantly greater number of likes and video views. When examining post characteristics that influenced the number of likes a post generated, posts that elicited negative emotions received $52.6 \%$ and $70.7 \%$ more likes than positive emotions $(P=.04)$ and neutral emotions $(P<.01)$, respectively. Upon assessment of posting characteristics that influenced the number of comments a post generated, promotional posts were shown to have $43.7 \%$ fewer comments than nonpromotional posts $(P=.02)$. When evaluating factors that influenced total number of interactions a post generated, it was found that posts that asked questions generated $26.4 \%$ more interactions $(P<.01)$ than those that do not. Conclusions: The present investigation found that the most effective strategies to generate more interactions on Instagram are those that elicit emotional responses and provoke viewer engagement by asking questions and directing actions. Additionally, it was found that promotional content was not well received by viewers. Clinical Relevance: Orthopaedic surgeons have an opportunity to connect with colleagues, patients, and interested viewers through social media platforms in order to enhance their practice, disseminate educational content, and contribute to the social media presence of orthopaedic surgery.
\end{abstract}

\section{Introduction}

A merican adults have seen a drastic increase in cellphone ownership from $62 \%$ in 2002 to $96 \%$ in 2019. ${ }^{1}$ Currently, 7 in 10 Americans use social media to connect with one another, engage with news content, share information, and entertain themselves. Instagram is a prominent social media platform where users share photos and videos with a worldwide audience. $^{2}$ Originally used for keeping in contact with friends and acquaintances, the social media conglomerate has taken over as a place for product marketing,

Henry Ford Hospital, Department of Orthopaedic Surgery, Detroit, Michigan, U.S.A. (M.J.A., L.S.K.); Wayne State University School of Medicine, Detroit, Michigan, U.S.A. (A.H., M.E.D., G.D.); and Mayo Clinic, Department of Orthopedic Surgery, Minneapolis, Minnesota, U.S.A. (K.R.O).

The authors report the following potential conflicts of interest or sources of funding: K. R. Okoroha reports hospitality payments from Stryker and Zimmer and educational support from Arthrex and Smith $\theta$ Nephew, outside the submitted work. Full ICMJE author disclosure forms are available for this article online, as supplementary material. keeping up with current events, and even receiving informative content on a wide variety of educational content. The use of social media, Instagram especially, is expanding the field of health care as well. Medical professionals use the platform for public-directed marketing and peer-to-peer education, which would have required newspaper advertisements and brochure handouts in years past. Instagram has been used to document occupation-related experiences and for some, has subsequently resulted in microcelebrity status; a stature that has become recognized as a niche

Received January 27, 2021; accepted May 28, 2021.

Address correspondence to Lafi S. Khalil, M.D., Department of Orthopaedic Surgery 2799 W. Grand Blvd., Detroit, MI 48202,U.S.A. E-mail: lskhalil@ gmail.com

(C) 2021 THE AUTHORS. Published by Elsevier Inc. on behalf of the Arthroscopy Association of North America. This is an open access article under the CC BY-NC-ND license (http://creativecommons.org/licenses/by-nc-nd/4.0/). 2666-061X/21102

https://doi.org/10.1016/j.asmr.2021.05.011 
area of interest. ${ }^{3}$ The utility of social media in the medical field became further conspicuous during the novel severe acute respiratory syndrome coronavirus 2 (or SARS-CoV-2) virus pandemic. During the pandemic, health information from multiple regional, national, and international health authorities, regulating bodies, and professional associations were frequently shared across social media platforms as quickly as the landscape was changing. ${ }^{4}$

As social media platforms have changed the landscape of information distribution, it is important to understand which information is gaining the greatest audience, while healthcare professionals adapt with the new communication norms of this era. The term "influencer" was officially added to the English dictionary in 2019..$^{5}$ A "social media influencer" is a person or brand who has a substantial digital footprint in their online community, as defined by platform, and whose opinions drive thoughts and trends within that community. ${ }^{6}$ Through influencers, the public receives free and accessible information and recommendations about a variety of topics. This has transformed "influencer marketing" into a fast-growing billion-dollar industry. ${ }^{5}$ The social media platform does not, however, validate the credibility of information shared by independent users and influencers. Although theoretically physicians and medical professionals would be the most ideal influencers in health care, building a following and badge of credibility is intricate and timeconsuming. On the other hand, a void of information opens the door for less reliable sources to influence viewership. As a response to this need of reliable health communication, many physicians have turned to social media to bridge the gap. In conjunction with being a reliable source of evidence-based information, these physicians also have the opportunity to promote their practices, connect with patients, discuss the latest technological advances and procedures, and provide educational content.

Given the constitutive nature of social media, physician influencers have the potential to be a formidable resource of medical information. However, growing a social media presence and following requires strategically created content and a connection with viewership. By understanding elements of successful social media engagement, dissemination of accurate health information by physicians and other health care professionals may become more effective. There is a paucity of information regarding the elements needed to promote orthopaedic surgery-based social media platforms. The purpose of this study is to analyze the Instagram practices of current orthopaedic surgeons and the components associated with highest reach and interactions. The hypothesis was that the most effective strategies used by orthopaedic surgeons to generate more interactions on Instagram would be to elicit emotional responses and provoke viewer engagement through posting strategies, such as asking questions and directing actions.

\section{Methods}

\section{Design}

A retrospective content analysis was performed using a mixed-methods study design, validated by previously published investigations, to analyze public Instagram posts by orthopaedic surgeons worldwide. ${ }^{7-9}$ The association between the highest viewed content posted by orthopaedic surgeon influencers and the audience interactions was examined to better understand the components shared by successful social media influencers. Instagram profiles were selected for inclusion if they belonged to orthopaedic surgeons of any subspecialty and if they used the hashtag \#ortho. When this search was conducted, the hashtag \#ortho was selected because it was the most popular orthopaedic surgeryrelated hashtag on the platform, with more than half a million posts using the hashtag. Additionally, using the hashtag \#ortho enabled the search to capture all orthopaedic related hashtags, including \#orthopedic, \#orthopaedic, \#orthopaedicsurgery, \#orthopedicsurgery, etc. The top 25 orthopaedic surgeon Instagram accounts using this hashtag were identified by the number of Instagram followers and the top 10 post, based on number of likes, were examined. The top 25 orthopaedic surgeon Instagram profiles were identified using a social media analysis marketing tool (Heepsy, Inc., Bilbao, Spain). The top 25 orthopaedic surgeon Instagram profiles using the hashtag \#ortho were ranked by the number of followers. Account information regarding followers, posts, engagement percentage, average likes, average comments, average video view, average video likes, average video comments, and estimated cost per post were all provided through Heepsy. Accounts were aggregated on the basis of which region in the world the account holder is from, the subspecialty of the account holder, and the engagement rating of the account. In order to increase the sample size and create roughly equally sized cohorts, continent of origin was aggregated into North America, Asia, and Other. When evaluating accounts on the basis of the subspecialty, all accounts were aggregated into three categories: Sports, Trauma, and Others. This aggregation was determined on the basis of the subspecialties that were represented by the top 25 orthopaedic surgeons using \#ortho on their posts; additionally, this allowed for three roughly equal cohorts. When assessing account information based on engagement ratings, accounts were divided into low $(0 \%$ to $1 \%)$, average ( $1 \%$ to $2.5 \%)$, and high $(+2.5 \%)$. 
Engagement percentage is equal to the sum of comments and likes that an account has, divided by the number of followers, and multiplied by 100. The aggregation of engagement rating was based on industry standards and established by a social media analysis marketing tool (Heepsy, Inc., Bilbao, Spain) used in this study. An analysis of each Instagram profiles' top 10 posts was conducted. Post data were collected using the social media marketing tool Popsters, Inc., in July 2020. Popsters provided the top 10 posts determined by the number of Instagram likes only for each account. Each Instagram post in the top 10 was then analyzed for content, tone, interactions from the audience, and relationship building techniques by the surgeon, as defined below.

\section{Data Collection}

The review of Instagram posts was performed by two individual observers and any discrepancies were clarified by mutual agreement. Quantitative data collected included Instagram engagement between the surgeon and their audience. Interactions for each post are defined as the sum of the total number of likes plus the total number of comments. Quantitative data also included identifying the use of 1) a real-world tie-in and 2) the use of promotional content. Qualitative data collected include the format of the post, content of the post, prompting engagement from the surgeon, relationship-building techniques used, the emotion induced by the post, the type of real-world tie-in used if any, the type of promotional content if used, and the overall tone of post (Appendix). The format of each post was labeled as either photo, video, or text. The categories for content of the post were limited to a categorical choice of lifestyle, radiology, operating room images, education, family, a professional self-image. If the content did not fit into one of the defined categories, it was labeled as "other". Prompting engagement was defined as asking a question or asking the audience to complete an action. Posts that did not fit in either category were labeled as none. Relationship-building techniques were denoted as either prompting a reply from the audience in the comments or posting about relatable content. Posts were either labeled as one or the other, both, or neither. The emotion variable had the categories of positive, negative, or neutral. For the variable real-world tie-in, after the quantitative assessment of yes or no was established, we further investigated the post to determine what the tie-in was. The same methodology used for real-world tie-in was implemented for categorizing the use of promotional content in the post. The final qualitative variable investigated was the tone of the post. Instagram posts were labeled as either positive, negative, optimistic, serious, or neutral, based on predetermined criteria (Appendix).

\section{Statistical Analysis}

All continuous data are reported as means \pm standard deviation, while categorical data are reported as counts and column percentages [N (\%)]. For continuous variables, group comparisons are performed using KruskalWallis tests due to low group counts and non-normal distributions of the variables. Pairwise comparisons are performed using the Dwass, Steel, Crithlow-Fligner (DSCF) method for continuous variables. For categorical variables, group comparisons are performed using $\chi^{2}$ tests when expected cell counts are $>5$ and using Fisher's exact tests when expected cell counts are $<5$. To see how the characteristics of each Instagram post relate to the number of likes, comments, and total interactions, mixed effect models were fit using maximum likelihood methods. Statistical significance is set at $P<.05$. All analyses are performed using SAS 9.4 (SAS Institute, Inc., Cary, NC).

\section{Results}

\section{Account Information}

The top 25 Orthopaedic Surgery Instagram accounts using the \#ortho were identified. Overall, accounts had an average of $517.9 \pm 326.6$ posts, $116,400.0 \pm$ $136,434.1$ followers, and $2.0 \% \pm 1.9 \%$ engagement percentage. Descriptive analysis of all accounts is provided in Table 1. Distribution of all accounts based on followers is provided in Figure 1. There was no statistically significant difference between the number of posts, followers, engagement percentage, average likes, average comments, estimate cost per post, average video views, average video likes, average video comments, estimated cost per video, or comment-to-like

Table 1. Account Information for the Top 25 Accounts Using the Hashtag Ortho

\begin{tabular}{lcc}
\hline & Mean & Standard Deviation \\
\hline Number of posts & 517.96 & 326.58 \\
Number of followers & $116,400.00$ & $136,434.05$ \\
Engagement percentage & $2.01 \%$ & $1.97 \%$ \\
Likes & $2,468.33$ & $3,676.20$ \\
Comments & 46.79 & 57.48 \\
Video views & $10,020.00$ & $1,543.83$ \\
Video likes & $1,968.00$ & $3,322.95$ \\
Video comments & 60.86 & 94.97 \\
Subspecialties & & \\
$\quad$ Sports & 8 & \\
Trauma & 8 & \\
Other & 9 & \\
Continent of origin & & \\
$\quad$ North America & 7 & \\
Asia & 11 & \\
Other & 7 & \\
Engagement rating & & \\
$\quad$ Low & 10 & \\
Average & 8 & \\
High & 7 & \\
\hline
\end{tabular}




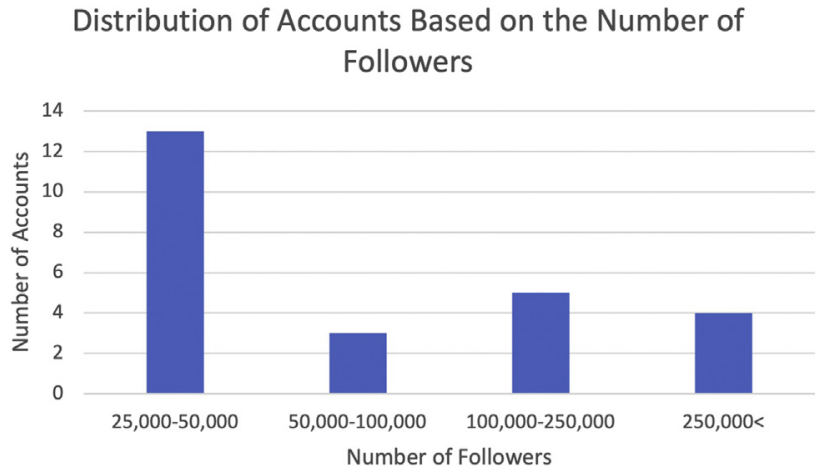

Fig 1. The distribution of included orthopaedic surgeon Instagram accounts based on the number of followers.

ratio based on continent of origin $(P>.05)$. No significant difference was present between subspecialties for any metric $(P>.05)$. Increased engagement rating was significantly related to the likes, views, and costs per post (Table 2). Accounts with increased engagement rating had a significantly greater number of likes $(6,200.0 \pm 4,949.4$ vs $531.1 \pm 898.6$ and $1,382.5 \pm$ $1,544.9, P<.01)$, video views $(30,750.0 \pm 17,017.1$, $1002.9 \pm 1307.3$, and $6060.0 \pm 6973.7$ vs, $P<.01)$, and average cost a sponsor would pay the account holder for an image $(562.2 \pm 422.5$ vs $86.6 \pm 105.2$ and $257.3 \pm 232.7$ dollars, $P<.01$ ) or for a video $(3,512.5 \pm 1,775.0$ vs $287.2 \pm 276.4$ and $1,208.0 \pm$ $1,264.7, P=.02$ ) post (Table 2).

\section{Factors Influencing Receiving Likes}

The top 10 posts from each account were determined on the basis on number of likes. Among the 250 posts evaluated, the average number of likes was $8,367.0 \pm$ $13,248.4$, average number of comments was $121.0 \pm$ 211.5 , and the average number of total interactions was $8,488 \pm 13,349.5$. Regression models were used to access which post characteristics, format, content, engagement, relationship, emotion, real world tie-in, promotional, and tone, influenced the total number of likes, total number of comments, and total number of interactions (Table 3). The number of likes a post received was significantly related to the engagement method and emotion elicited $(P=.04$ and $P=.01$, respectively). Posts that both asked questions and elicited an action received $25.1 \%$ more likes compared to those that did not use either engagement strategy (beta estimate (standard error), .22 (.08), $P<.01)$. Asking questions alone or provoking an action alone did not have a significant impact on the total likes a post had $(P=.61$ and $P=.11$, respectively). Posts received $52.6 \%$ and $70.7 \%$ more likes when eliciting negative emotions rather than positive emotions (beta estimate (standard error), -.75 (.35), $P=.04$ ) and neutral emotions (beta estimate [standard error], -1.23 [.34], $P<.01)$, respectively.

\section{Factors Influencing Receiving Comments}

The number of comments generated on a post was significantly influenced by the post format, emotion elicited, tone, and the presence of promotional content $(P<.01, P=.01, P=.02$, and $P<.01$, respectively $)$ (Table 4 ). Photo posts on Instagram were expected to generate $66.8 \%$ less comments than video posts (beta estimate (standard error), $-1.10(.28), P<.01)$. There was no significant association between the total comments received and the emotion elicited by a post (negative vs positive emotions; $P=.16$ ), unless it was a neutral post, which received $74.3 \%$ fewer comments overall (beta estimate [standard error], $-1.4[.51], P=$ .02 ). The tone of the post (positive, optimistic, neutral, or serious) did not significantly impact the number of comments generated $(P=.87, P=.96, P=.10$, and $P=$ .57, respectively). Promotional posts were demonstrated to have $43.7 \%$ less comments than nonpromotional posts (beta estimate [standard error], -.57 [.24], $P=.02)$.

\section{Influence on Total Interaction}

Engagement method and emotions elicited were found to have significant correlations with the total

Table 2. Influence of Account Engagement on Posting Trends

\begin{tabular}{|c|c|c|c|c|}
\hline & Low $(n=10)$ & Average $(n=8)$ & High $(n=7)$ & $P$ Value \\
\hline 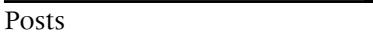 & $539.00(354.29)$ & $464.88(391.05)$ & $548.57(233.13)$ & .6458 \\
\hline Followers & $111500(166690)$ & $91375.0(110849)$ & $152000(126845)$ & .3322 \\
\hline Engagement percentage & $.49(.42)$ & $1.80(.96)$ & $4.43(1.87)$ & $<.0001$ \\
\hline Average likes & $531.11(898.62)$ & $1382.50(1544.92)$ & $6200.00(4949.41)$ & .001 \\
\hline Average comments & $21.78(39.91)$ & $37.38(36.70)$ & $89.71(75.92)$ & .0575 \\
\hline Estimated cost per post & $86.63(105.17)$ & $257.31(232.68)$ & $562.21(422.53)$ & .0084 \\
\hline Average views per video & $1002.86(1307.25)$ & $6060.00(6973.68)$ & $30750.0(17017.1)$ & .0073 \\
\hline Average likes per video & $165.71(115.85)$ & $1045.60(855.71)$ & $6275.00(4525.02)$ & .0062 \\
\hline Average comments per video & $15.00(7.11)$ & $32.60(34.66)$ & $153.50(145.39)$ & .2772 \\
\hline Estimated cost per video & $287.20(276.35)$ & $1208.00(1264.69)$ & $3512.50(1775.00)$ & .0242 \\
\hline Comment-to-like ratio & $3.30(2.45)$ & $3.81(2.26)$ & $1.67(1.83)$ & .1443 \\
\hline
\end{tabular}

Bolded values indicate significant difference $(P<.05)$. 
Table 3. Regression Model for Factors Influencing Number of Likes

\begin{tabular}{lccc}
\hline \multicolumn{1}{c}{$\begin{array}{c}\text { Beta Estimate } \\
\text { (Standard } \\
\text { Error) }\end{array}$} & $\begin{array}{c}\text { Expected } \\
\text { Percent } \\
\text { Change }\end{array}$ & $P$ Value \\
\hline Format & & & .4817 \\
Content & & & .9403 \\
Engagement & (reference $=$ none) & & $\mathbf{. 0 4 0 2}$ \\
Action & $.115(.072)$ & $12.79 \%$ & .1109 \\
Questions & $-.083(.163)$ & $-7.96 \%$ & .6092 \\
Both & $.224(.084)$ & $25.11 \%$ & $\mathbf{. 0 0 8 3}$ \\
Relationship & & & .5554 \\
Emotion & (reference $=$ negative) & & $\mathbf{. 0 0 1 1}$ \\
Positive & $-.747(.347)$ & $-52.62 \%$ & $\mathbf{. 0 4 3 3}$ \\
Neutral & $-1.228(.343)$ & $-70.71 \%$ & $\mathbf{. 0 0 1 8}$ \\
Real World & & & .2712 \\
$\quad$ Tie-In & & & .7719 \\
Promotional & & & .0612 \\
Tone & & & \\
\hline
\end{tabular}

Bolded values indicate significant difference $(P<.05)$.

number of interactions a post generates $(P=.03$ and $P=$ $<.01$, respectively) (Table 5). When compared to posts that did not use any engagement strategies, posts that asked questions were expected to generate $26.4 \%$ more interactions (beta estimate [standard error], .23 [.09], $P$ $<.01)$. Posts that elicited actions and used questions combined did not see a significant increase in expected interactions. Posts that elicited an emotion demonstrated a significant relationship with the total number of interactions $(P<.01)$, with positive or neutral emotions generating significantly less interactions when compared to negative posts (beta estimate [standard error], $-.75(.35), P=.04$ and $-1.23(.35)$, $P<.01$, respectively) .

Table 4. Regression Model for Factors Influencing Number of Comments

\begin{tabular}{lccc}
\hline & & & \\
\multicolumn{1}{c}{ Variable } & Beta Estimate & Percent & \\
Format & (Standard Error) & Change & $P$ Value \\
Photo & $-1.102(.281)$ & $-66.78 \%$ & $\mathbf{. 0 0 0 6}$ \\
Text & $-.960(.589)$ & $-61.71 \%$ & .1046 \\
Content & & & .8594 \\
Engagement & & & .6015 \\
Relationship & & & .5958 \\
Emotion & (reference $=$ negative) & & $\mathbf{. 0 0 9 8}$ \\
Positive & $-.077(.521)$ & $-7.41 \%$ & .1556 \\
Neutral & $-1.359(.514)$ & $-74.31 \%$ & $\mathbf{. 0 1 5 2}$ \\
Real World Tie-In & & & .5605 \\
Promotional & (reference $=$ no) & & $\mathbf{. 0 1 9 4}$ \\
Yes & $-.574(.244)$ & $-43.67 \%$ & $\mathbf{. 0 1 9 4}$ \\
Tone & (reference $=$ negative) & & $\mathbf{. 0 0 4 2}$ \\
Positive & $-.079(.475)$ & $-7.60 \%$ & .8683 \\
Optimistic & $-.025(.566)$ & $-2.47 \%$ & .9648 \\
Neutral & $-.851(.518)$ & $-57.30 \%$ & .1021 \\
Serious & $.277(.488)$ & $31.92 \%$ & .571 \\
\hline
\end{tabular}

Bolded values indicate significant difference $(P<.05)$.
Table 5. Regression Model for Factors Influencing Number of Interactions

\begin{tabular}{lccc}
\hline & & Expected & \\
\multicolumn{1}{c}{ Variable } & $\begin{array}{c}\text { Beta Estimate } \\
\text { (Standard Error) }\end{array}$ & $\begin{array}{c}\text { Percent } \\
\text { Change }\end{array}$ & $P$ Value \\
\hline Format & & & .3559 \\
Content & (reference $=$ none) & & .9648 \\
Engagement & $.126(.073)$ & $13.43 \%$ & .0312 \\
Action & $.234(.086)$ & $26.36 \%$ & $\mathbf{. 0 0 6 7}$ \\
Questions & $-.081(.165)$ & $-7.78 \%$ & .6285 \\
Both & & & .5007 \\
Relationship & (reference $=$ negative) & & $\mathbf{. 0 0 1}$ \\
Emotion & $-.752(.345)$ & $-52.86 \%$ & $\mathbf{. 0 4 0 8}$ \\
Positive & $-1.231(.341)$ & $-70.80 \%$ & $\mathbf{. 0 0 1 6}$ \\
Neutral & & & .262 \\
Real world tie-in & & & .8354 \\
Promotional & & & .0862 \\
Tone & &
\end{tabular}

\section{Discussion}

The present investigation found that among the orthopaedic surgeons with the greatest following, posts that elicited emotional responses, solicited viewer engagement in the form of asking questions and prompting actions, and used combinations of these strategies rather than in isolation, were successful in building a social media presence. Additionally, nonpromotional posts were found to generate significantly more comments than promotional content.

Patients have unlimited access to medical advice and expertise through various social media platforms, without formal validation or peer review of the posts generated. ${ }^{10}$ Often times, a social media account with a larger following carries greater clout and is perceived as disseminating more legitimate information. ${ }^{11}$ The use of viewer engagement strategies on social media platforms has become an increasingly common and effective way to interact with viewers and prompt their engagement, yet there is a paucity of literature evaluating this relationship among orthopaedic surgeon "influencers" on social media. In an evaluation of Facebook user engagement in health care-related posts, Willoughby et al. found a negative association with asking questions (beta $=-.10$ [.03], $P<.05) .{ }^{9}$ Likewise, prompting engagement by asking questions on diabetes mellitus-related social media posts led to a reduction in the times the post was shared or commented on in a study by Rus et al. (beta $=-1.19$ [.40] and beta $=.77[.31]$, respectively). ${ }^{12}$ Interestingly, the opposite was found true in this study as prompting engagement through the use of questions was associated with an increased number of total interactions (likes and comments). Additionally, posts that asked questions and prompted actions, such as telling the 
viewer to like and share the post, were significantly more likely to generate likes (beta $=.22$ [.08], $P<.01$ ). The stark contrast seen in the impact of engagement strategies in the present study is likely attributed to Instagram as the platform evaluated, as studies have shown that user engagement is $30-200$ times higher than it is for Facebook or Twitter. ${ }^{8}$ With this information, Orthopaedic Surgeons may be cognizant of engagement strategies employed on Instagram, such as creating polls, asking questions, and sharing posts. Additionally, these strategies may be less useful on Facebook or Twitter, platforms that may be better suited for informative or conversational content.

The emotional response generated by a post has been an element of interest in prior studies. In an analysis of social media strategies used by the food industry, Klassen et al. examined posting characteristics of 143 Facebook posts and 84 Instagram posts. ${ }^{7}$ Their study found that posts eliciting positive emotions had a significant relationship with the total number of interactions of Facebook posts (beta estimate [95\% CI], .31 [.04 to .57], $P=.02$ ), but not of Instagram posts (beta estimate [ $95 \% \mathrm{CI}], .47$ [ -.10 to 1.04$], P=.11$ ). In an evaluation of 165,000 political Twitter posts, Stieglitz et al. found that negative emotional responses elicited by tweets were more likely to be "retweeted," thereby enhancing dissemination of that information (beta coefficient $=.05, P<.05) .{ }^{13}$ The findings in the present study reproduce this phenomenon, demonstrating a significantly greater number of interactions for Instagram posts with negative emotions compared to those conveying positive or neutral emotions. Reaching a social media audience through posts eliciting negative emotions takes advantage of the underlying negativity bias that arises from normal psychosocial development. ${ }^{14}$ While the intended audience may gravitate to these types of posts, it is important to note that the information or content itself does not need to be negative or destructive, and that a delicate balance of emotions should be represented in posts to reach the greatest range of audience. One such post that was negative, but not destructive, in nature expressed frustration over the COVID-19 pandemic and the loss of a colleague. While the content is negative in nature the caption ended with a plea to everyone reading to do their part in keeping people safe during the pandemic. This exemplifies that negative content can still be used without promoting harmful or hostile content.

When evaluating the effects of promotional content on social media engagement, the literature is currently divided on the topic. Edney and colleagues examined health promotional campaigns on Facebook, Twitter, and Instagram, finding that product promotional posts had a significant and positive influence on post engagements $($ beta $=.24, P<.01) .{ }^{8}$ Likewise, Klassen et al. found that post engagement on both Facebook and Instagram increased with the use of promotional content in their analysis of the food industry (beta $=$ .81 [.50 to 1.13], $P<.001$ and beta $=1.32[.77$ to 1.88], $P<.001$, respectively). ${ }^{7}$ Conversely, in an investigation of the impacts of direct-to-consumer marketing on Facebook, Schultz et al. showed that promotional posts received the fewest "likes" (an indicator of engagement). ${ }^{15}$ The findings of the present study agree with Schultz and colleagues, indicating that promotional content had a significant negative association with the number of comments (i.e., engagement) a post generated. This may be explained by the similar "direct-to-consumer" relationship evaluated by Schultz et al., wherein an orthopaedic surgeon on Instagram is likely followed primarily by colleagues, patients, and viewers interested in the educational component of the account. Therefore, unlike food companies and consumer brand accounts, followers in the healthcare field are less likely to be engaged with promotional content.

\section{Limitations}

This investigation is not without limitations. One such limitation is inherent to the cross-sectional design of the study, as all data were collected during a single time point and for this reason cannot account for seasonal changes in posting trends. Additionally, account characteristics used were all provided by Heespy.com and might differ from those presented by other social media marketing analysis tools. The use of marketing analysis tools to gather account characteristics is not a validated method and is subject to inherent flaws in the marketing tool software; however, one advantage of using software is the elimination of human bias while assessing social media profiles. Furthermore, engagement metrics collected were limited to number of likes and comments since Instagram does not provide tools to evaluate the number of times a post has been shared via direct messaging or reposted or shared on Instagram live story. Because of the retrospective nature of this analysis, posts collected were limited to photo, text, and video posts. Instagram live stories were not included since they only appear on an account for 24 hours before being deleted, making it impossible to retrospectively track engagement with that type of post.

\section{Conclusion}

The present investigation found that the most effective strategies to generate more interactions on Instagram are those that elicit emotional responses and provoke viewer engagement through a number of posting strategies, such as asking questions and directing actions. Additionally, it was found that promotional content was not well received by viewers. 


\section{References}

1. Pew Research Center. Mobile fact sheet. vol. 2021. Available at, https://Www.pewresearch.org/internet/factsheet/mobile/ 2019. Accessed June 3, 2021.

2. Pew Research Center. Social media fact sheet. https:// Www.pewresearch.org/internet/fact-sheet/social-media/ 2019. Accessed June 3, 2021.

3. Kerr H, Booth R, Jackson K. Exploring the characteristics and behaviors of nurses who have attained microcelebrity status on Instagram: Content analysis. J Med Internet Res 2020;22:e16540.

4. Eghtesadi M, Florea A. Facebook, Instagram, Reddit and TikTok: A proposal for health authorities to integrate popular social media platforms in contingency planning amid a global pandemic outbreak. Can J Public Health 2020;111:389-391.

5. Brooks A. [Timeline] A brief history of influencers: Social media today. Available at, https://www.socialmediatoday. com/news/timeline-a-brief-history-of-influencers/554377/ 2019. Accessed June 3, 2021.

6. Blakemore JK, Bayer AH, Smith MB, Grifo JA. Infertility influencers: An analysis of information and influence in the fertility webspace. J Assist Reprod Genet 2020;37:1371-1378.

7. Klassen KM, Borleis ES, Brennan L, Reid M, McCaffrey TA, Lim MS. What people "like": Analysis of social media strategies used by food industry brands, lifestyle brands, and health promotion organizations on Facebook and Instagram. J Med Internet Res 2018;20:e10227.

8. Edney S, Bogomolova S, Ryan J, Olds T, Sanders I, Maher C. Creating engaging health promotion campaigns on social media: Observations and lessons from Fitbit and Garmin. J Med Internet Res 2018;20:e10911.

9. Card KG, Lachowsky N, Hawkins BW, Jollimore J, Baharuddin F, Hogg RS. Predictors of Facebook user engagement with health-related content for gay, bisexual, and other men who have sex with men: Content analysis. JMIR Public Health Surveill 2018;4:e38.

10. Dijkstra S, Kok G, Ledford JG, Sandalova E, Stevelink R. Possibilities and pitfalls of social media for translational medicine. Front Med (Lausanne) 2018;6:345.

11. Skoglösa N, Svelander E, Stedt L. Insta(nt) credibility? Exploring how Generation $\mathrm{Z}$ assigns credibility to influencers and their sponsored posts on Instagram. Jonkoping University 2019:70. Available at: http://hj.diva-portal.org/ smash/get/diva2:1320425/FULLTEXT01.pdf. Accessed June 3, 2021.

12. Rus HM, Cameron LD. Health communication in social media: Message features predicting user engagement on diabetes-related Facebook pages. Ann Behav Med 2016;50: 678-689.

13. Stieglitz S, Dang-Xuan L. Emotions and information diffusion in social media-Sentiment of microblogs and sharing behavior. J Mgmt Inform Syst 2013;29:217-248.

14. Vaish A, Grossmann T, Woodward A. Not all emotions are created equal: the negativity bias in social-emotional development. Psychol Bull 2008;134:383-403.

15. Schultz CD. Proposing to your fans: Which brand post characteristics drive consumer engagement activities on social media brand pages? Electr Commerce Res Applic 2017;26:23-34. 
Appendix Table 1. Framework Developed to Categorized Posting Strategies Used

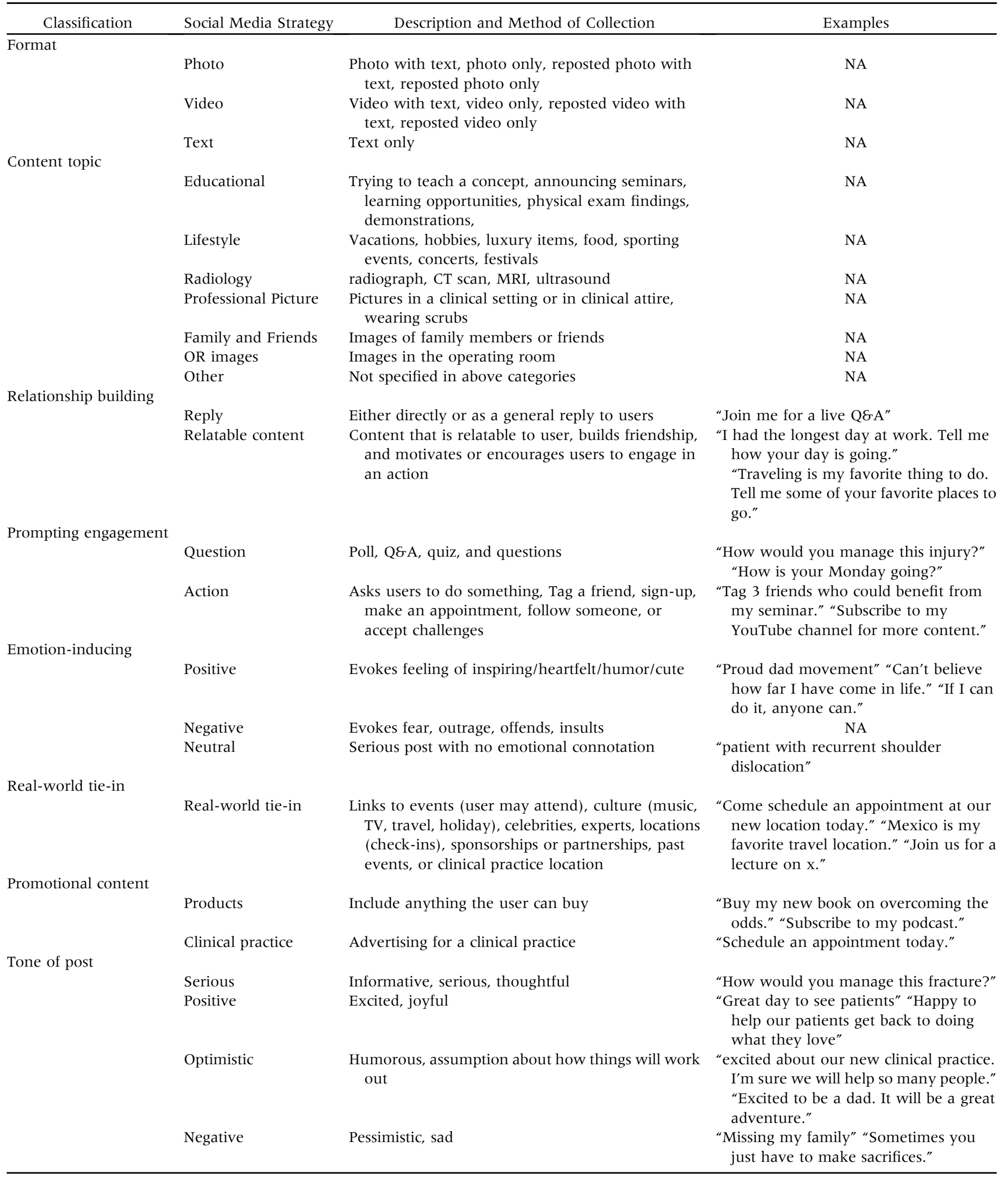

